\title{
Incidencia del Clima Organizacional y de las Actitudes Laborales en el Comportamiento Prosocial de los Profesionales de la Salud del Guayas Ecuador
}

\author{
María del C. Lapo-Maza(1) y Miguel A. Bustamante-Ubilla(2)* \\ (1) Pontificia Universidad Católica del Perú, Programa de Graduados de la Escuela de Negocios, \\ Av. Universitaria 1801, Lima 32, Perú. (e-mail: maria.lapo@pucp.pe) \\ (2) Universidad de Talca, Programa de Investigación de Excelencia Interdisciplinaria en Envejecimiento \\ Saludable (PIEI-ES), Dos Norte 685, 3465548 Talca, Chile. (e-mail: mabu@utalca.cl) \\ * Autor a quien debe ser dirigida la correspondencia
}

Recibido Dic. 21, 2017; Aceptado Mar. 12, 2018; Versión final Abr. 26, 2018, Publicado Oct. 2018

\begin{abstract}
Resumen
El presente trabajo muestra cómo las dimensiones clima organizacional y actitudes laborales inciden en el comportamiento prosocial. Se aplicaron tres cuestionarios, debidamente autorizados por sus respectivos autores, todos estructurados en escala Likert de 5 niveles. Se aplicó el método de encuesta a una muestra aleatoria de 583 trabajadores de hospitales. Se realizó análisis de factores exploratorio, se modeló la incidencia mediante ecuaciones estructurales y se confirmaron las relaciones mediante análisis de único mediador. Los resultados del análisis de primer orden indican que el clima organizacional incide en forma directa sobre la variable mediadora actitudes laborales y ésta en forma directa sobre comportamiento prosocial. Además, se determinar que existe un efecto indirecto del clima organizacional sobre el comportamiento prosocial. El análisis de único mediador confirma que actitudes laborales actúan como mediadora en la relación clima organizacional respecto de comportamiento prosocial.
\end{abstract}

\section{Organizational Climate Incidence and Labor Attitudes in the Prosocial Behavior of Guayas (Ecuador) Health-Care Professionals}

\begin{abstract}
The present work shows how the dimensions organizational climate and labor attitudes impact in the prosocial behavior. Three questionnaires were chosen, properly authorized by their respective authors, all structured in a 5 levels Likert scale. The survey method was applied to a random sample of 583 hospitals workers. An exploratory analysis of factors was done, the incidence was modeled by means of structural equations and the relationships were confirmed by means of the single mediator's analysis. The results of a first order analysis indicate that the organizational climate impacts directly on the mediator labor attitudes and this variable has a direct impact on prosocial behavior. It is also determined that an indirect effect of the organizational climate exists on the prosocial behavior. The single mediator's analysis confirms that labor attitudes act as mediator of the relationship between the organizational climate and the prosocial behavior.
\end{abstract}

Keywords: organizational climate; labor attitudes; behavior prosocial; exploratory factorial analysis; structural equations 


\section{INTRODUCCIÓN}

En los años 60, a partir del fenómeno conocido como efecto del espectador (Moñivas, 1996) se investigó las actitudes buscando explicar por qué las personas ante una situación de emergencia, piensan que alguien va a ayudar a la persona necesitada pero, como todos piensan lo mismo, ninguno le brinda socorro (Latané y Darley, 1970). Fue así como con el transcurso del tiempo, se generó una hipótesis de conducta que implicaba indiferencia y hasta indolencia que dio pie a toda una línea de investigación de la conducta de ayuda en contextos interpersonales (Arias, 2015) ahora denominada conducta o comportamiento prosocial (Ackfeldt y Wong, 2006). El estudio del comportamiento prosocial (CPS) en las organizaciones se remonta a Katz y Kahn (1966) para explicar conductas discrecionales que van más allá de los requisitos formales del trabajo (Bülbül, 2014). Este comportamiento en su perspectiva organizacional, ha sido llamado comportamiento extra-rol (Vandyne et al., 1995) o de desempeño contextual y ha sido estudiado en relación con los estados emocionales de compromiso afectivo, valores e intereses compartidos que impactan la satisfacción y la motivación de los empleados (Bernal et al., 2015). El CPS, analiza la predisposición de los empleados de ir más allá de los requisitos formales del trabajo (Ackfeldt y Wong, 2006; Kanten, 2014) en beneficio de terceros (Bülbül, 2014), y es la expresión de un esfuerzo individual que satisface alguna meta personal y reporta calidad de vida (Das y Vijayalakshml, 2013). Sin embargo, los empleados necesitan de alguna fuerza impulsora de apoyo organizacional y estilo de liderazgo (Kanten, 2014) que los sostenga en dicha actitud laboral positiva (Ackfeldt y Wong, 2006).

La multidimensionalidad del clima organizacional (Bustamante et al., 2016; Juárez-Adauta, 2012) puede otorgar esa fuerza impulsora que colabora a que directivos y trabajadores comprendan y den sentido a su realidad. Por una parte, porque un mejor clima organizacional (Bernal et al., 2015) motiva las actitudes laborales positivas y la participación social y por otra, porque activa el comportamiento prosocial de los trabajadores (Ackfeldt y Wong, 2006). El CPS prescribe, en consecuencia, un extra-rol de cooperación (Vandyne et al., 1995) significativo y motivacional para los empleados que impacta sus conductas laborales (Malhotra y Ackfeldt, 2016) y estimula internamente el sistema de relaciones (Mendoza-Sierra et al, 2014). Las actitudes laborales se definen entonces a partir de la influencia del clima organizacional que genera una predisposición al comportamiento (Ackfeldt y Wong, 2006) que estimula el compromiso con la organización e impulsa actitudes positivas que van más allá de su trabajo (Kanten, 2014). Es por ello que las actitudes laborales adquieren un carácter mediador de las estrategias de gestión y del clima organizacional que progresivamente redefinen el comportamiento prosocial (Vandyne et al., 1995). Así mismo, se ha demostrado que a largo plazo el CPS se integra a la cultura y se expresa en normas de reciprocidad, cohesión de grupo, oportunidades de crecimiento y desarrollo de capacidades (Bülbül, 2014), lo que es particularmente relevante en las instituciones prestadoras de servicios de salud, porque las actitudes laborales positivas (Patterson, et al., 2004) influyen en el comportamiento, incrementa la satisfacción laboral y mejoran la calidad del servicio entregado a los clientes (Mendoza-Sierra et al, 2014).

En consecuencia el CPS tiene efectos organizacionales que impiden al sector de la salud sustraerse de la necesidad de conocer cómo los individuos ajustan sus actitudes laborales y la manera como se expresan éstas en el servicio al paciente y como los trabajadores de la salud van más allá de sus roles laborales y dan forma a lo que se denomina comportamientos prosociales en beneficio de los demás, sean estos sus propios pares o bien usuarios y pacientes (Hyde, et al., 2013). Es por esto que algunos investigadores estiman que los trabajadores de la salud pueden ser altruistas (Smith, et al., 2013) puesto que el medio de trabajo, sus funciones y responsabilidades, les permiten expresar conductas prosociales que son valoradas por la sociedad, primero, porque su trabajo lo desarrollan en un ambiente complejo y, segundo, porque su desempeño puede tener consecuencias relevantes sobre las personas y la comunidad. Sobre la base de los principales conceptos analizados y sus diversas interrelaciones, el presente trabajo pretende determinar la incidencia del clima organizacional y las actitudes laborales sobre el comportamiento prosocial, en el contexto de los profesionales del sector de la salud de la provincia del Guayas, Ecuador.

\section{METODOLOGÍA}

El presente trabajo cuantitativo y transversal, adopta primero un carácter exploratorio para validar los reactivos de los constructos en estudio (Baño, 2016; Kaplan, 2000), luego confirmatorio para corroborar la estructura de factores y finalmente, mediante análisis de un único mediador, para ratificar cómo las dimensiones de los constructos independientes y mediadores actúan sobre los factores dependientes (Freiberg, et al. 2013), puestos en relación de acuerdo con la teoría debidamente referenciada.

\section{Población y muestra}

Para definir el tamaño de la muestra se analizaron las características de los instrumentos, el número de ítems por factor, escalas de respuesta, homogeneidad de la muestra y comunalidad de los ítems (Freiberg, 
et al. 2013). Además, se verificaron los criterios de muestra mínima (n) y de proporción $(\mathrm{n} / \mathrm{p} ; 10: 1 ; 5: 1)$ de encuestados por número de ítems determinándose un alcance mínimo de 500 observaciones para dar solidez al estudio. (Lloret et al., 2014)

La muestra, para una p oblación finita (Freiberg, et al. 2013), se determinó mediante un análisis de poder usando el programa G*Power 3.1 (Faul et al., 2009). Este análisis arrojó un tamaño mínimo de la muestra de 504 trabajadores considerando un alfa de Cronbach $(\alpha=0,05)$, tamaño del efecto $(T E=0,20)$, una potencia $(1-\beta=0,95)$.

\section{Procedimiento}

La provincia del Guayas elegida para el desarrollo del presente trabajo es la más poblada de Ecuador (INEC, 2015), cuenta con una amplia cobertura de servicios de salud y fue propuesta al programa de doctorado dependiente de CENTRUM de la Pontificia Universidad Católica de Lima, Perú.

Se eligió como marco muestral hospitales de mediana y alta complejidad, privados y públicos y como elementos a médicos, enfermeras y auxiliares de enfermería, representativos del sector de la salud (Freiberg, et al. 2013).

El trabajo de campo fue realizado por entrevistadores debidamente entrenados con una duración promedio de respuesta fue de 30 a 40 minutos. El levantamiento de los datos se realizó entre los meses de marzo y mayo de 2017. Los cuestionarios fueron aplicados de acuerdo con las normativas de anonimato y confidencialidad establecidas por CENTRUM, presentando el consentimiento informado a los entrevistados.

\section{Instrumentos de investigación}

Los cuestionarios fueron elegidos primero, considerando la reciente aplicación de los mismos. El de clima organizacional aplicado el año 2016 (Bustamante et al., 2016), el de Actitudes laborales aplicado el año 2013 y el de comportamiento prosocial aplicado los años 2014 y 2016 (Malhotra y Ackfeldt, 2016). En segundo lugar, los tres cuestionarios cuentan con escalas de medición idénticas, Likert, de cinco niveles (1 $=$ Totalmente en desacuerdo hasta $5=$ Totalmente de acuerdo) y, se obtuvo los permisos de uso otorgados por los respectivos autores.

Para los tres constructos se verificaron los índices KMO, GFI, AGFI, CFI, NNFI, alpha ( $\alpha$ ) de Cronbach, omega ( $(\Omega)$ McDonald's y RMSEA (Chión y Charles, 2016; Freiberg, et al. 2013). Además, los cuestionarios originales escritos en idioma inglés, fueron traducidos al español mediante el método de doble traducción. Luego se sociabilizaron con un grupo de expertos, académicos y directivos de servicios de salud, a fin de ratificar textos, semántica y expresiones de los reactivos.

El cuestionario de clima organizacional diseñado por Bustamante, et al., (2016), cuenta con 44 reactivos agrupados en diez dimensiones: (1) colaboración y buen trato, (2) motivación laboral, (3) iniciativa participativa, (4) desarrollo profesional, (5) apoyo al emprendimiento, (6) equipo y distribución de personas y material, (7) organización y desempeño, (8) estabilidad laboral, (9) comunicación y desarrollo y (10) autonomía y profesionalismo.

El cuestionario de actitudes laborales diseñado por Ackfeldt y Wong (2006), contiene 14 reactivos agrupados en dos dimensiones: (1) compromiso afectivo organizacional y (2) satisfacción laboral.

Finalmente, el cuestionario de comportamiento pro-social propuesto por Ackfeldt y Wong (2006), registra 21 ítems condensados en tres dimensiones: (1) comportamiento pro-social esperado y deseable, (2) comportamiento pro-social extra rol y (3) cooperación.

\section{Hipótesis del estudio}

Dado que la gestión de los factores que conforman el clima organizacional (Bernal et al., 2015) motiva la participación social y activa el comportamiento prosocial de los trabajadores (Ackfeldt y Wong, 2006), se formula la primera hipótesis de investigación.

H1: Que el clima organizacional tiene un efecto directo sobre el comportamiento prosocial, en el contexto de los profesionales de la salud del Guayas, Ecuador.

Además, considerando por otra parte, que las dimensiones del clima organizacional (Bernal et al., 2015; Bustamante et al., 2016) influyen sobre las dimensiones que definen las actitudes de las personas y que éstas modelan su comportamiento al interior de las organizaciones se define la segunda hipótesis de investigación. 
H2: Que el clima organizacional influye en forma directa sobre las actitudes laborales, en el contexto de los profesionales de la salud del Guayas, Ecuador.

Por último, a partir del hecho de que el clima organizacional da sentido a la realidad laboral (Bustamante et al., 2016; Juárez-Adauta, 2012) e influye las actitudes laborales (Choi y Joung, 2017) y que estas últimas, generan una predisposición a un comportamiento prosocial de los individuos (Malhotra y Ackfeldt, 2016), el presente trabajo define la tercera hipótesis de investigación.

H3: Que las actitudes laborales influyen en forma directa y como mediadora entre el clima organizacional y el comportamiento prosocial, en el contexto de los profesionales de la salud del Guayas, Ecuador.

Análisis factorial previo de los constructos

De acuerdo con la metodología de análisis previo sugerida por Baño (2016) y Kaplan (2000), se aplicó análisis paralelo (PA) considerando la matriz de dispersión policórica (Freiberg, et al. 2013), análisis robusto de los mínimos cuadrados no ponderados (RULS) y rotación oblicua (oblimin directo), con el objetivo de asumir la eventual interdependencia de factores latentes que pudiesen resultar correlacionados. Además, considerando que los tres cuestionarios miden las percepciones mediante escalas policóricas de 5 puntos, se puede asumir que dicha escala colabora a superar los supuestos de no normalidad de los ítems (Lloret et al., 2014).

Así mismo, de acuerdo con la metodología de (Freiberg, et al. 2013), se optó por exigir a los reactivos comunalidades altas $(\geq 0,5)$ para asegurar correlaciones fuertes entre ítems y constructos (Bandalos y Finney, 2010) y se exigieron cargas factoriales superiores $(\geq 0,6)$ a fin de excluir reactivos escasamente correlacionados y asegurar una correcta interpretación de las estimaciones multivariantes (Kaplan, 2000).

Se revisó la unidireccionalidad de las escalas y se verificó el número de ítems mínimo ( $\geq 3)$ para cada constructo (Freiberg, et al. 2013). Además, se evaluó la medida de adecuación $\operatorname{KMO}(\geq 0,80)$, los coeficientes de significancia de Bartlett y se analizó la varianza explicada para obtener la configuración más simple de los constructos (Lloret et al., 2014).

\section{Modelamiento de ecuaciones estructurales (SEM)}

Se trabajó con las variables observables de los tres constructos definidas como policóricas (Freiberg, et al. 2013), se caracterizaron como variables latentes endógena, mediadora y exógenas para diseñar el modelo de ecuaciones estructurales y se aplicó el método máxima verosimilitud, ML. Además, con el objetivo de verificar resultados se aplicó el método Mínimos Cuadrados no ponderados, ULS y, finalmente, para confirmar las relaciones, se aplicó el método de único mediador (MacKinnon y Dwyer, 1993).

El ajuste iterativo de las variables observadas se realizó mediante el análisis de los índices de modificación y se verificaron los índices de bondad de ajuste. Para ello se aplicaron los índices Estadístico-Radio de Verosimilitud de Chi-Cuadrado (CMIN/DF $\leq 3$ ), el de variabilidad explicada por el modelo, GFI $(\geq 0,9)$, el error de aproximación cuadrático medio, $\operatorname{RMSEA}(\leq 0,05)$ y el índice de error cuadrático medio, RMR $(\approx 0)$ (Freiberg, et al. 2013).

Complementariamente se verificaron los índices ajustado de bondad, AGFI $(\leq 0,8)$, el no normado de ajuste, TLI $(\leq 0,9)$ equivalente a NNFI y la desviación estándar de las diferencias entre los valores pronosticados y los valores observados, RMSR denominado también, desviación media cuadrática (RMSD) o error cuadrático medio (RMSE) (Chión y Charles, 2016).

\section{Modelo de único mediador}

Finalmente, se aplicó el modelo de único mediador de MacKinnon y Dwyer (1993) para comprobar la mediación de la variable actitudes laborales, utilizando el enfoque de los pasos causales (Baron y Kenny, 1986; Judd y Kenny, 1981).

El método de validación discriminante (Henseler, et al., 2015) requirió las pruebas de significancia separadas. a) la relación de la variable independiente con la variable moderadora, mediante el estimados a; b) la relación de la variable moderadora sobre la variable independiente, a través del estimados b; c) la variable independiente en relación a la variable dependiente sin el efecto de la variable moderadora, por medio del estimados $\mathrm{c}$ y d) la variable independiente en relación a la variable dependiente considerando el efecto de la variable moderadora, mediante el estimador c' (MacKinnon y Fairchild, 2009).

Se establecieron tres dimensiones principales, las de clima organizacional que consolida 29 ítems, la mediadora de actitudes laborales que resume 14 reactivos y las dependientes de comportamiento prosocial 
que totaliza 19 ítems, con estos agregados se calculó la sumatoria de frecuencias generando variables cuantitativas continuas (Freiberg, et al. 2013).

\section{RESULTADOS}

A continuación se detallan los resultados de los análisis exploratorios AFE, confirmatorio AFC, modelamiento estructural SEM y método de único mediador.

\section{Análisis de la muestra}

La muestra inicial alcanzó un total de 629 contactos. De los entrevistados, 276 eran médicos, 173 licenciadas en enfermería y 180 auxiliares en enfermería. Sin embargo, con el objetivo de eliminar registros perdidos o casos extremos, se aplicó análisis de diagrama de caja de SPSS obteniéndose una muestra depurada de 583 casos. Esta muestra, superior a la determinada a priori ( $\geq 504$ casos), fue redefinida mediante análisis post hoc $G^{*}$ Power 3.1 que sugirió, dado un alfa de Cronbach $(\alpha=0,05)$, una potencia $(1-\beta$ $=0,975)$ y tamaño de efecto $=0.2$.

La estructura de edades de la muestra, en su mayor proporción, se ubica en los rangos de 26 - 34 años (29.4\%) y entre los 35 - 43 años (23.1\%). La mayoría es de sexo femenino $(70 \%)$.

\section{Análisis exploratorio de los constructos}

Considerando que los tres constructos provienen de estudios internacionales efectuados en diversas realidades socio culturales, se analizaron ítems, factores, confiabilidad, adecuación y significancia (Freiberg, et al. 2013) para corroborar si los reactivos de cada uno de los instrumentos se ratifican en el contexto de los profesionales de la salud del Guayas, Ecuador (Tabla 1).

El cuestionario de clima organizacional con 44 reactivos y 10 factores, fue sensibilizado mediante el análisis de comunalidades y cargas factoriales. Se excluyeron 15 reactivos, confirmando el modelamiento de 29 ítems agrupados en cuatro factores. Los factores rediseñados FC1, FC2 y FC3 muestran una varianza explicada significativa de $35.1 \%$ ** $17.7 \%$ ** y $6,4 \%^{*}$ respectivamente y $\mathrm{FC} 4$, una varianza explicada de $5,5 \%$, no significativa, con una varianza real acumulada de $64.7 \%$. Los índices de este instrumento fueron, KMO de 0.925, GFI de 0.989, AGFI de 0.985, CFI de 0.961, NNFI de 0.947, a de Cronbach de 0.903 , un $\Omega$ McDonald's de 0.821, un RMSR, de 0.041y un RMSEA de 0.082 .

El constructo actitudes laborales con 14 reactivos mantuvo sus ítems validando sus componentes mediante los índices de comunalidad y cargas factoriales. Los reactivos sensibilizados se agruparon en dos factores FA1 con una varianza explicada de $72,9 \%^{* *}$ significativa y FA2 con una varianza explicada de $8,3 \%$, no significativa; totalizando una varianza acumulada de $81.2 \%$. Los indicadores del cuestionario muestran un KMO de 0.954, GFI de 0.999, un AGFI de 0.999, un CFI de 0.999, un NNFI de 1.000, un a de Cronbach de 0.963 , un $\Omega$ McDonald's de 0.963 , un RMSR es de 0.049 y un RMSEA de 0,086. De los 21 ítems de comportamiento prosocial (Tabla 1), se eliminaron 2 reactivos quedando 19 ítems agrupados en dos factores, FP1 con una varianza explicada de $62,9 \%^{* *}$ y FP2 que muestra una varianza explicada de $7,8 \%$ no significativa, sumando un $70,7 \%$ de varianza total explicada. Finalmente, los índices de este último constructo entregan un KMO de 0.9643, GFI de 0.994, un AGFI de 0.993, un CFI de 0.984, un NNFI de 0.980 , un a de Cronbach de 0.965 , un ' $\Omega$ McDonald's de 0.965 , un RMSR de 0.0195 y un RMSEA de 0.084 .

\section{Modelamiento confirmatorio de las variables observadas}

Para confirmar la estructura interna de los factores determinados mediante AFE, se realizó el modelamiento de los tres constructos mediante ecuaciones estructurales por el método de máxima verosimilitud, ML (Freiberg, et al. 2013).

Tabla 1: Ítems y factores del análisis exploratorio de los constructos clima organizacional, actitudes laborales y comportamiento prosocial

\begin{tabular}{|c|c|c|c|c|c|c|c|c|c|c|}
\hline \multicolumn{6}{|c|}{ Clima Organizacional } & \multicolumn{2}{|c|}{ Actitudes Laborales } & \multicolumn{3}{|c|}{ Comportamiento Prosocial } \\
\hline VAR. & FC1 & $\mathrm{FC} 2$ & FC3 & FC4 & VAR. & FA1 & FA2 & VAR. & FP1 & FP2 \\
\hline V 1 & 0.773 & & & & V 45 & & 0.702 & V 59 & & 0.844 \\
\hline V 2 & 0.741 & & & & V 46 & & 0.739 & V 60 & & 0.841 \\
\hline V 3 & 0.779 & & & & V 47 & & 0.796 & V 61 & & 0.792 \\
\hline
\end{tabular}


Tabla 1 (continuación)

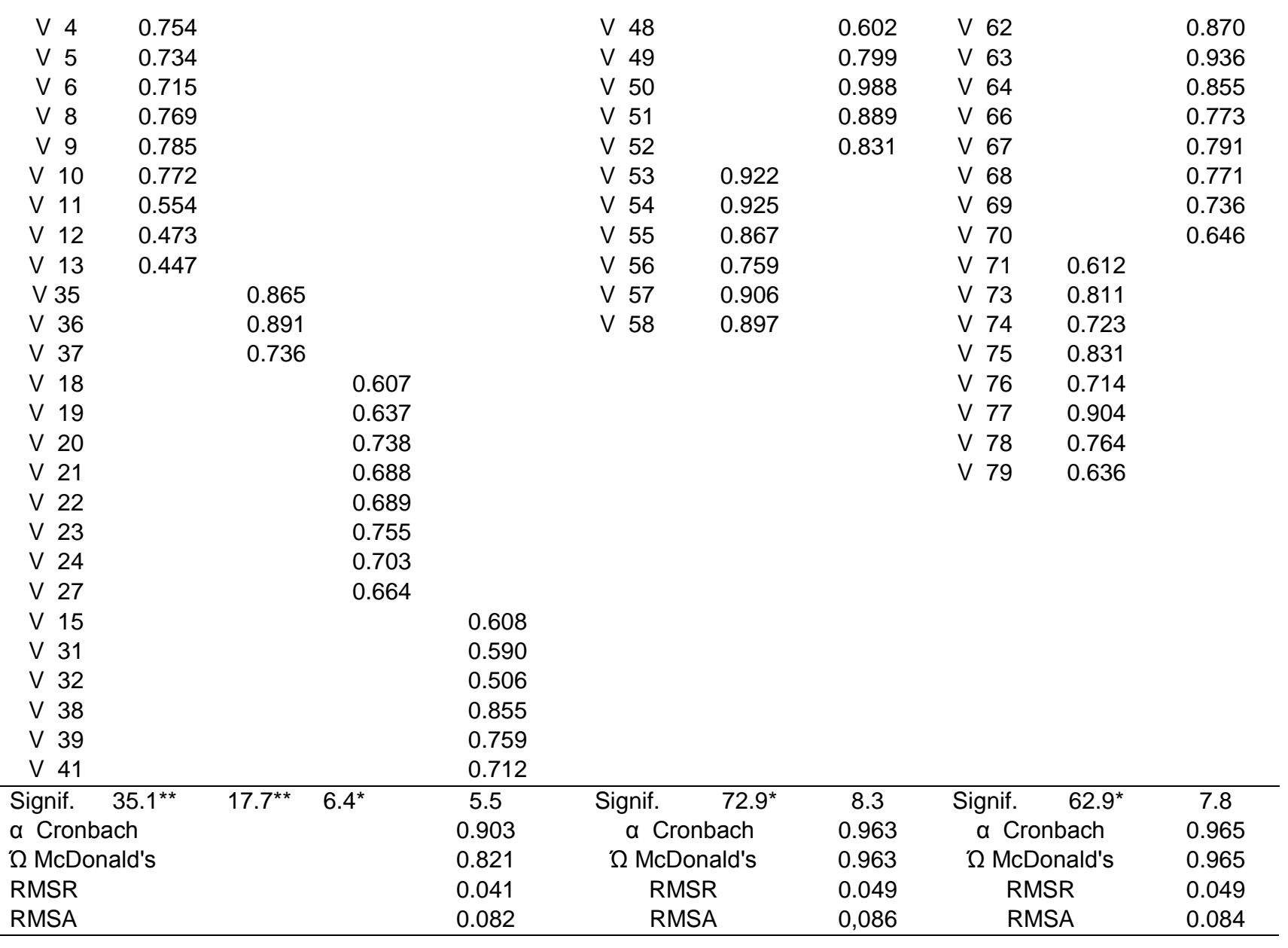

La tabla 2, presenta los índice regresión $\beta_{1}$ e índice estandarizado de varianza del error $\beta_{2}$, de cada una las ecuaciones de los reactivos que conforman las dimensiones analizadas. La fórmula utilizada se muestra en la expresión (1).

$Y_{n}=\beta_{1} F P i+\beta_{2}$

Donde; $\mathrm{n}$ : Variable observada, $\mathrm{B}_{1}$ : Varianza de la variable con su factor, FPi: Factor al que pertenece la variable y $\beta_{2}$ : varianza del error de cada variable

Se observa que los índices $\beta_{1}$ y $\beta_{2}$ muestran índices estándar con valores positivos perfectamente ajustados al rango que va entre 0 y 1 , indicando que el modelo se ajusta adecuadamente, presentando significación al $1 \%$ en todos los ítems.

Los índices significativos al 1\% indican que los ítems de cada uno de los factores que conforman los constructos de clima organizacional, actitudes laborales y comportamiento prosocial, fueron debidamente ratificados y agrupados en sus respectivos factores. Además, la significación estadística señala que los reactivos validados confirman su relación con su respectivo factor.

\section{Especificación del modelo}

Se definieron como variables latentes exógenas los cuatro factores de clima organizacional (FC), como dimensiones mediadoras los dos factores de actitudes laborales (FA) y como dimensiones endógenas los dos factores de comportamiento prosocial (FP).

Las variables latentes independientes de clima organizacional son FC1: gestión interna, FC2: estabilidad laboral, FC3: tensión interna y desarrollo profesional, FC4: organización y desempeño. Las variables latentes mediadora de actitudes laborales son FA1: satisfacción laboral y FA2: compromiso afectivo. Las variables latentes dependientes de comportamiento prosocial son FP1: cooperación y FP2: calidad y servicio al paciente. 
Tabla 2: Parámetros del modelo de medida por el método ML

\begin{tabular}{|c|c|c|c|c|c|c|c|}
\hline \multicolumn{4}{|c|}{ Clima Organizacional } & \multirow[b]{2}{*}{$\begin{array}{c}\text { Variables y } \\
\text { error }\end{array}$} & \multirow[b]{2}{*}{$\begin{array}{l}\beta_{1} \text { Índice } \\
\text { Regresión }\end{array}$} & \multicolumn{2}{|c|}{ Actitudes Laborales } \\
\hline $\begin{array}{l}\text { Variables y } \\
\text { error }\end{array}$ & $\begin{array}{l}\beta_{1} \text { Índice } \\
\text { Regresión }\end{array}$ & $\begin{array}{c}\text { Índice } \\
\text { Estandarizado }\end{array}$ & $\begin{array}{c}\beta_{2} \\
\text { Varianza } \\
\text { del Error }\end{array}$ & & & $\begin{array}{c}\text { Índice } \\
\text { Estandarizado }\end{array}$ & $\begin{array}{c}\varepsilon_{1} \beta_{2} \\
\text { Varianza } \\
\text { del Error }\end{array}$ \\
\hline \multicolumn{4}{|c|}{ Gestión interna } & \multicolumn{4}{|c|}{ Satisfacción laboral } \\
\hline $\begin{array}{l}\mathrm{V} 11-\mathrm{eV} 11 \\
\mathrm{~V} 12-\mathrm{eV} 12 \\
\mathrm{~V} 13-\mathrm{eV} 13 \\
\mathrm{~V} 14-\mathrm{eV} 14 \\
\mathrm{~V} 15-\mathrm{eV} 15\end{array}$ & $\begin{array}{l}1,000^{\star \star *} \\
1,109^{\star \star \star} \\
1,148^{\star \star *} \\
1,241^{\star \star \star} \\
1,107^{\star \star \star}\end{array}$ & $\begin{array}{l}0,675 \\
0,745 \\
0,751 \\
0,814 \\
0,725\end{array}$ & $\begin{array}{l}0,611^{\star * *} \\
0,503^{\star \star \star} \\
0,521^{* \star *} \\
0,401^{\star \star \star} \\
0,565^{\star \star \star}\end{array}$ & $\begin{array}{l}\text { V68 - eV68 } \\
\text { V67 - eV67 } \\
\text { V66 - eV66 } \\
\text { V65 - eV65 } \\
\text { V64 - eV64 } \\
\text { V63 - eV63 }\end{array}$ & $\begin{array}{l}1,000^{\star * *} \\
0,933^{\star * *} \\
0,969^{\star * *} \\
0,996^{* * *} \\
0,991^{* \star *} \\
0,941^{\star * *} \\
\end{array}$ & $\begin{array}{l}0,895 \\
0,899 \\
0,816 \\
0,927 \\
0,882 \\
0,826 \\
\end{array}$ & $\begin{array}{l}0,154^{\star * *} \\
0,127^{\star * *} \\
0,290^{\star * *} \\
0,099^{\star * *} \\
0,173^{\star * *} \\
0,254^{\star * *}\end{array}$ \\
\hline V16-eV16 & $1,203^{\star * *}$ & 0,779 & $0,481^{\star * *}$ & \multicolumn{4}{|c|}{ Compromiso afectivo } \\
\hline $\mathrm{V} 18-\mathrm{eV} 18$ & $0,923^{\star \star *}$ & 0,646 & $0,607^{\star \star *}$ & V62 - eV62 & $1,000^{* \star *}$ & 0,873 & $0,226^{\star * *}$ \\
\hline $\mathrm{V} 19-\mathrm{eV} 19$ & $0,913^{* * *}$ & 0,654 & $0,570^{\star \star *}$ & V61 - eV61 & $1,039^{* * *}$ & 0,894 & $0,196^{\star * *}$ \\
\hline V20 - eV20 & $0,942^{\star * \star}$ & 0,658 & $0,594^{* * *}$ & V60 - eV60 & $1,079^{* * *}$ & 0,922 & $0,148^{\star \star *}$ \\
\hline V21 - eV21 & $1,074^{\star \star *}$ & 0,717 & $0,557^{* \star *}$ & V59 - eV59 & $0,999 * * *$ & 0,867 & $0,239^{* * *}$ \\
\hline V22 - eV22 & $1,979^{* * *}$ & 0,633 & $0,733^{* * *}$ & V58 - eV58 & $0,577^{\star * *}$ & 0,505 & $0,705^{\star \star *}$ \\
\hline V23 - eV23 & $1,067^{\star \star *}$ & 0,666 & $0,731^{\star \star \star}$ & V57 - eV57 & $0,853^{\star * *}$ & 0,727 & $0,469^{\star * *}$ \\
\hline & & & & V56 - eV56 & $0,843^{* * *}$ & 0,700 & $0,536^{\star * *}$ \\
\hline & & & & V55 - eV55 & $0,947^{\star \star *}$ & 0,721 & $0,600^{* * *}$ \\
\hline \multicolumn{4}{|c|}{ Estabilidad laboral } & \multicolumn{4}{|c|}{ Comportamiento Pro-social } \\
\hline V45-eV45 & $1,000^{\star \star *}$ & 0,869 & $0,363^{\star * \star}$ & & B1 Índice & Índice & $\beta_{2}$ \\
\hline V46 - eV46 & $0,971^{\star * *}$ & 0,890 & $0,277^{\star \star *}$ & & Rearesión & monce & Varianza \\
\hline $\mathrm{V} 47-\mathrm{eV} 47$ & $0,881^{* * *}$ & 0,757 & $0,651^{* * *}$ & & & Estandarızado & del Error \\
\hline \multicolumn{4}{|c|}{ Tensión interna y desarrollo profesional } & \multicolumn{4}{|c|}{ Cooperación } \\
\hline V37 - eV37 & $1,000^{* * *}$ & 0,621 & $1.001^{* * *}$ & V81-eV81 & $1,000^{* * *}$ & 0,216 & $1,669^{* \star *}$ \\
\hline V34 - eV34 & $1,116^{\star * *}$ & 0,732 & $0,677^{* * *}$ & V83 - eV83 & $2,462^{* * *}$ & 0,822 & $0,239^{* * *}$ \\
\hline V33 - eV33 & $0,977^{* * *}$ & 0,716 & $0,571^{* * *}$ & V84 - eV84 & $2,388^{* * *}$ & 0,830 & $0,211^{* * *}$ \\
\hline V32-eV32 & $0,899^{* * *}$ & 0,637 & $0,745^{\star * *}$ & V85 - eV85 & $2,579 * * *$ & 0,838 & $0,231^{* * *}$ \\
\hline V31 - eV31 & $1.169^{* * *}$ & 0,769 & $0,592^{\star \star *}$ & V86 - eV86 & $2,532^{* * *}$ & 0,889 & $0,139^{* * *}$ \\
\hline V30 - eV30 & $1,171^{\star * *}$ & 0,773 & $0,579^{* * *}$ & V87 - eV87 & $2,328^{* * *}$ & 0,770 & $0,305^{\star * *}$ \\
\hline V29 - eV29 & $1.007^{* \star *}$ & 0,658 & $0,832^{\star * *}$ & V88 - eV88 & $2,352^{* * *}$ & 0,794 & $0,265^{\star * *}$ \\
\hline V28 - eV28 & $0,867^{\star \star \star}$ & 0,612 & $0,788^{\star * \star}$ & V89 - eV89 & $2,459^{* * *}$ & 0,863 & $0,170^{* * *}$ \\
\hline \multicolumn{4}{|c|}{ Organización y desempeño } & \multicolumn{4}{|c|}{ Calidad y servicio al paciente } \\
\hline & & & & V69 - eV69 & $1.000^{* * *}$ & 0,739 & $0,303^{* \star *}$ \\
\hline & & & & V70 - eV70 & $1,013^{\star \star *}$ & 0,760 & $0,273^{\star \star \star}$ \\
\hline & & & & V71 - eV71 & $1,032^{\star * \star}$ & 0,744 & $0,314^{* * *}$ \\
\hline V51-eV51 & $1,000^{\star \star \star}$ & 0,773 & $0,506^{\star \star \star *}$ & V72 - eV72 & $1,071^{\star * *}$ & 0,859 & $0,148^{* * *}$ \\
\hline V49 - eV49 & $1,012^{* * *}$ & 0,782 & $0,487^{* *}$ & V73 - eV73 & $1,073^{\star \star *}$ & 0,857 & $0,152^{\star * *}$ \\
\hline V48 - eV48 & $0,998^{* *}$ & 0,736 & $0,632^{\star \star \star}$ & V74 - eV74 & $1,013^{\star * *}$ & 0,779 & $0,244^{\star * *}$ \\
\hline V42 - eV42 & $0,761^{\star * *}$ & 0,647 & $0,604^{* * *}$ & V76 - eV76 & $1,079^{* * *}$ & 0,820 & $0,207^{* * *}$ \\
\hline V41 - eV41 & $0,978^{\star \star *}$ & 0,756 & $0,539^{* * *}$ & & $1,101^{\text {** }}$ & 0,875 & $0,135^{\star \star *}$ \\
\hline V25 - eV25 & $0,948^{* * *}$ & 0,709 & $0,668^{* * *}$ & V78 - eV78 & 1,090 *** & 0,858 & $0,155^{\text {***}}$ \\
\hline & & & & V79 - eV79 & $1,107^{\star * *}$ & 0,860 & $0,157^{\star \star *}$ \\
\hline & & & & V80 - eV80 & $1,119^{\text {** }}$ & 0,836 & $0,196^{\star * *}$ \\
\hline
\end{tabular}

\section{Determinación del modelo estructural}

Con el objetivo de ajustar los tres constructos, se analizaron las relaciones de covarianza entre las dimensiones de clima organizacional y relaciones de varianza entre las dimensiones de clima, actitudes laborales y de comportamiento prosocial.

\section{Análisis de covarianzas}

Se observa en la Tabla 3, que las relaciones de covarianza muestran, en general, índices bajos cercanos a 0 y no significativos entre las dimensiones de clima organizacional, lo que podría indicar que dichos factores se comportan de manera independiente, como es el caso de la relación entre tensión interna y desarrollo profesional y gestión interna ( $F C 1<->F C 3,-0,006)$ la que actúa en forma inversa aunque no significativa. Se observan sin embargo dos excepciones que establecen relaciones altas y significativas. En la primera excepción se observa la dimensión latente gestión interna, que muestra una relación natural sobre organización y desempeño ( $F C 1<->F C 4,0,484^{* * \star}$ ) actuando en forma directa y significativa al $1 \%$ y en ambos sentidos entre los factores. Por otro lado, en la segunda excepción se observa la relación entre el factor tensión interna y desarrollo profesional que incide de manera directa y significativa también al $1 \%$ sobre el factor estabilidad laboral (FC2 <-> FC3, 0,361**) 
Esta relación entre estabilidad laboral y tensión interna y desarrollo profesional, dado el contexto ecuatoriano en el que se desarrolla la encuesta, puede estar inducido por el hecho que la búsqueda de una mayor estabilidad laboral, puede hacer surgir al interior de las organizaciones, mayor competencia interna por mejorar la posesión de cargos y, por esta causa afectar el ambiente e incluso, estimular la tensión interna en la organización.

Ahora bien, visto de manera inversa, cuando una empresa mejora de manera interna las capacidades de sus trabajadores, se asume que existe mayor estabilidad laboral, se percibe una gestión positiva de la tensión interna y desarrollo profesional, lo que consolida las percepciones de estabilidad laboral.

Tabla 3: Estimadores de los Factores

\begin{tabular}{|c|c|c|c|c|c|}
\hline \multicolumn{6}{|c|}{ Relación de Covarianzas } \\
\hline \multirow{2}{*}{ Relaciones } & \multicolumn{2}{|c|}{$M L$} & \multirow{2}{*}{ Relaciones } & \multicolumn{2}{|c|}{$M L$} \\
\hline & Ajustado & Estándar & & Ajustado & Estándar \\
\hline $\mathrm{FC} 1<->\mathrm{FC} 2$ & 0,037 & 0,048 & FC2 $<->$ FC3 & $0,361^{* * *}$ & 0,430 \\
\hline FC1 $<->$ FC3 & -0.006 & -0.010 & FC2 $<->$ FC4 & 0,046 & 0,050 \\
\hline FC1 $<->$ FC4 & $0,484^{\star * *}$ & 0,781 & FC3 <-> FC4 & -0.014 & -0.020 \\
\hline \multicolumn{6}{|c|}{ Relación de Varianzas } \\
\hline $\mathrm{FA} 1 \leftarrow \mathrm{FC} 1$ & 0,065 & 0,059 & $\mathrm{FP} 2 \leftarrow \mathrm{FC} 3$ & 0,020 & 0,027 \\
\hline $\mathrm{FA} 1 \leftarrow \mathrm{FC} 2$ & $0,152^{\star \star *}$ & 0.205 & $\mathrm{FP} 2 \leftarrow \mathrm{FC} 4$ & $-0,143^{* *}$ & $-0,204$ \\
\hline $\mathrm{FA} 1 \leftarrow \mathrm{FC} 3$ & 0,001 & 0,001 & $\mathrm{FP} 2 \leftarrow \mathrm{FA} 1$ & $0,508^{* * *}$ & 0,660 \\
\hline $\mathrm{FA} 1 \leftarrow \mathrm{FC} 4$ & $0,459^{\star * \star}$ & 0,506 & $\mathrm{FP} 2 \leftarrow \mathrm{FA} 2$ & $0,098^{* *}$ & 0,138 \\
\hline $\mathrm{FA} 2 \leftarrow \mathrm{FC} 1$ & 0,062 & 0,052 & $\mathrm{FP} 1 \leftarrow \mathrm{FA} 2$ & $0,072^{* * *}$ & 0,213 \\
\hline $\mathrm{FA} 2 \leftarrow \mathrm{FC} 2$ & $0,187^{\star \star *}$ & 0.234 & $\mathrm{FP} 1 \leftarrow \mathrm{FA} 1$ & $0,181^{* * *}$ & 0,496 \\
\hline $\mathrm{FA} 2 \leftarrow \mathrm{FC} 3$ & 0,016 & 0.015 & $\mathrm{FP} 1 \leftarrow \mathrm{FC} 4$ & $-0,063^{\star *}$ & $-0,190$ \\
\hline $\mathrm{FA} 2 \leftarrow \mathrm{FC} 4$ & $0,565^{\star \star *}$ & 0,576 & $\mathrm{FP} 1 \leftarrow \mathrm{FC} 3$ & $-0,023$ & 0,064 \\
\hline $\mathrm{FP} 2 \leftarrow \mathrm{FC} 1$ & $0,190^{\star * *}$ & 0,225 & $\mathrm{FP} 1 \leftarrow \mathrm{FC} 2$ & $-0,009$ & 0,032 \\
\hline $\mathrm{FP} 2 \leftarrow \mathrm{FC} 2$ & 0,008 & 0.014 & $\mathrm{FP} 1 \leftarrow \mathrm{FC} 1$ & $0,113^{\star *}$ & 0,282 \\
\hline
\end{tabular}

Análisis de varianzas

La tabla 3 muestra además, que la dimensión independiente de clima organizacional gestión interna incide sobre cooperación $\left(\mathrm{FP} 1 \leftarrow \mathrm{FC} 1,0,113^{* *}\right.$ ) y sobre calidad y servicio al paciente $\left(\mathrm{FP} 1 \leftarrow \mathrm{FC} 1,0,190^{* * *}\right.$ ) en forma directa y significativa (5\%). De igual manera, el factor de clima estabilidad laboral incide en forma directa y significativa al $1 \%$ sobre los factores mediadores satisfacción laboral (FA1 $\leftarrow$ FC2, $0,152^{* * *}$ ) y compromiso afectivo (FA2 $\leftarrow \mathrm{FC} 2,0,187^{* * *}$ ).

Por su parte el factor organización y desempeño, se relaciona con las dimensiones mediadoras satisfacción laboral $\left(\mathrm{FA} 1 \leftarrow \mathrm{FC} 4,0,459^{\star * *}\right.$ ) y compromiso afectivo (FA2 $\leftarrow \mathrm{FC} 4,0,565^{\star * \star}$ ) en forma directa y significativa estableciendo un efecto que resulta en ambos casos evidente dado que una adecuada gestión de la organización y su desempeño interno propicia reacciones positivas en los factores mediadores. Sin embargo, organización y desempeño se relaciona a su vez con los factores de comportamiento prosocial cooperación (FP1 $\leftarrow$ FC4, $-0,063^{\star *}$ ) y calidad y servicio al paciente (FP2 $\leftarrow$ FC4, $-0,143^{\star *}$ ) mostrando efectos inversos y significativos al $5 \%$. Esta relación inversa del factor organización y desempeño sobre las dimensiones de comportamiento prosocial, pueden estar ocasionadas por el nivel de exigencia interno que la organización requiera de sus trabajadores, en cuyo caso, los esfuerzos y concentración de estos, no les permiten eventualmente transferir adecuadamente sus servicios hacia terceros, sean estos sus pares en el trabajo o clientes.

Por último, la dimensiones mediadoras de actitudes laborales satisfacción laboral y compromiso afectivo inciden en forma directa sobre los factores de comportamiento prosocial cooperación y calidad y servicio al paciente, en todos los casos en forma directa y significativa al 1\%. Esta última relación pone en evidencia que las actitudes laborales, como factores mediadores, activan positivamente los comportamientos prosociales a causa de sí mismos como también porque transfieren los efectos del factor organización y desempeño de clima organizacional. 


\section{Modelo estructural de las variables mediadoras y dependientes}

La Tabla 4 muestra los valores estandarizados $(0-1)$ del modelo estructural de las dimensiones mediadoras y dependientes. Las expresiones (2) y (3) muestran los coeficientes significativos al $1 \%$ de los factores independientes de clima organizacional que inciden en forma directa sobre las variables mediadoras de actitudes laborales.

$$
\begin{aligned}
& F A 1=0,152 F C 2+0,459 F C 4+0,395 \\
& F A 2=0,187 F C 2+0,565 F C 4+0,395
\end{aligned}
$$

Como se observa, el factor independiente de clima organizacional que más influye sobre los factores mediadores de actitudes laborales satisfacción laboral y compromiso afectivo, es organización y desempeño alcanzando índices de 0,459 y 0,565 respectivamente. Este efecto tiene mucho sentido en la gestión de organizaciones complejas del sector de salud, por cuanto su logística, complejidad técnica y lo impredecible de los requerimientos sociales, se resuelve precisamente mediante una adecuada gestión del factor organización y desempeño.

Le sigue el factor estabilidad laboral que incide también en forma directa $(0,152$ y 0,187$)$ sobre los factores de actitudes laborales, estableciendo una relación natural de efectos positivos por cuanto la estabilidad en el trabajo activa sentimientos de satisfacción y compromiso de los trabajadores para con su organización.

Por otra parte, los coeficientes significativos al $1 \%$ y al $5 \%$ de las ecuaciones estructurales de los factores endógenos de comportamiento prosocial cooperación y calidad y servicio al paciente se presentan en las expresiones (4) y (5).

$$
\begin{aligned}
& F P 1=0,113 F C 1-0,063 F C 4+0,181 F A 1+0.072 F A 2+0.043 \\
& F P 2=0,190 F C 1-0,143 F C 4+0,508 F A 1+0,098 F A 2+0,167
\end{aligned}
$$

\begin{tabular}{|c|c|c|c|c|c|c|}
\hline \multirow[t]{2}{*}{ Factor } & \multicolumn{2}{|c|}{ FP1 Cooperación } & \multicolumn{2}{|c|}{ FP2 Calidad y Servicio al Cliente } & \multirow{2}{*}{ Error FP1 } & \multirow{2}{*}{ Error FP2 } \\
\hline & No estándar & Estandarizado & No estándar & Estandarizado & & \\
\hline FC1 & $0,113^{\star \star \star}$ & 0,282 & $0,190 * * *$ & 0,225 & 0,043 & 0,167 \\
\hline FC4 & $-0,063^{* *}$ & $-0,190$ & $-0,143^{\star *}$ & $-0,204$ & & \\
\hline FA1 & $0,181^{* * *}$ & 0,496 & $0,508^{* * *}$ & 0,660 & & \\
\hline FA2 & $0,072^{* \star *}$ & 0,213 & $0,098^{* *}$ & 0,138 & & \\
\hline
\end{tabular}

Tabla 4: Estimadores significativos del modelo estructural

Dimensiones dependientes de comportamiento prosocial

Dimensiones mediadoras de actitudes laborales

\begin{tabular}{ccccccc}
\hline Factor & \multicolumn{2}{c}{ FA1 } & \multicolumn{2}{c}{ FA2 } & Error FA1 & Error FA2 \\
\hline & No estándar & Estandarizado & No estándar & Estandarizado & & 0,395 \\
FC2 & $0,152^{* * *}$ & 0,205 & $0,187^{* * *}$ & 0,234 & 0,395 \\
FC4 & $0,458^{* * *}$ & 0,506 & $0,565^{* * *}$ & 0,576 & & \\
\hline
\end{tabular}

Analizada la incidencia del clima sobre el comportamiento prosocial, se observa que gestión interna inciden sobre los factores cooperación y calidad y servicio al paciente de manera directa y significativa al $5 \%$ y $1 \%$ respectivamente. Por su parte, organización y desempeño, incide levemente pero de manera inversa y significativa al $5 \%$, sobre los factores de comportamiento prosocial.

Verificada además, la incidencia de clima sobre las actitudes laborales, el factor organización y desempeño incide en forma directa y significativa al $1 \%$ sobre las dimensiones de actitudes laborales mientras que el factor estabilidad laboral incide en forma directa y significativa al 1\% sobre los factores mediadores satisfacción laboral y compromiso afectivo, en consecuencia, el clima organizacional inciden sobre las actitudes laborales. 
Interpretando la incidencia de los factores de actitudes laborales sobre el comportamiento prosocial, satisfacción laboral y compromiso afectivo, inciden en forma directa y significativa al $1 \%$ y $5 \%$ sobre los factores cooperación y calidad y servicio al paciente.

La figura 1, muestra el modelamiento de los factores independientes de clima organizacional, los factores mediadores de actitudes laborales y las dimensiones dependientes de comportamiento prosocial.

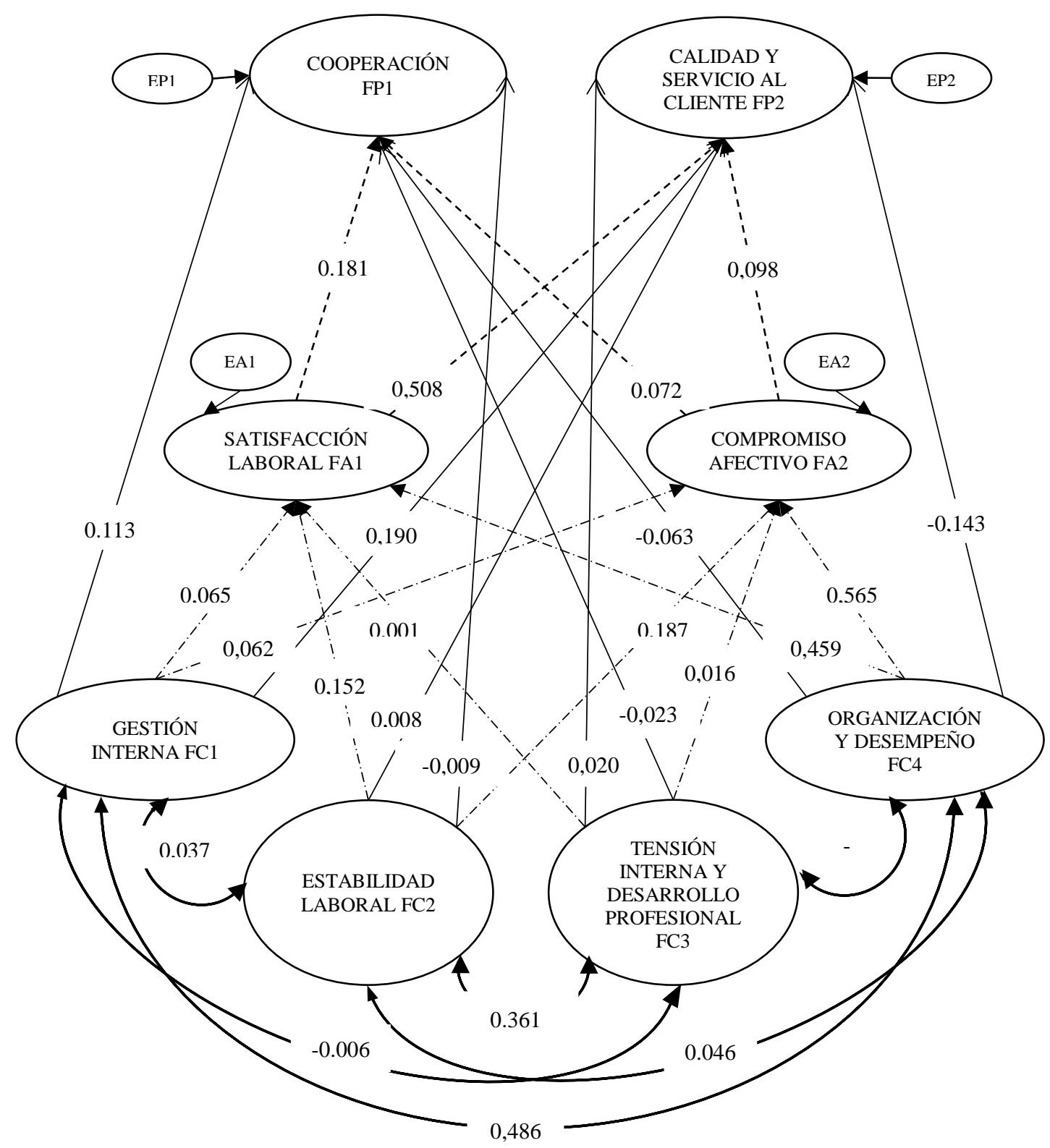

Fig. 1: Estimadores de relación de los factores

\section{Bondad de ajuste del modelo}

Los índices de bondad de ajuste se determinaron por el método ML y mediante el análisis de los índices de modificación, además los índices se comprobaron con la aplicación del método ULS (Tabla 5).

Se observa en general, que los índices de bondad de ajuste se ubican en rangos cercanos a los óptimos, por lo que se sostiene adecuadamente el modelamiento planteado.

En particular, los coeficientes CMIN/DF entregan valores alrededor de los 2 puntos $(\leq 3)$ comprobando el ajuste del modelo propuesto. Además la sumatoria del error de aproximación cuadrático medio RMSEA alcanza un valor de 0,047 $(\leq 0,05)$, lo que se considera muy bueno. 
Tabla 5: Bondad de ajustes de los modelos ML y ULS aplicados

\begin{tabular}{|c|c|c|c|c|}
\hline \multicolumn{5}{|c|}{ Mediante varianzas } \\
\hline $\begin{array}{l}\text { Índice de } \\
\text { Bondad }\end{array}$ & ML sin Ajuste & ML Ajustado & ULS sin Ajuste & ULS Ajustado \\
\hline CMIN/DF & 3,609 & 2,289 & 2,295 & 2,031 \\
\hline CMIN & 6506,402 & 4000,697 & 4137,284 & 3550,634 \\
\hline DF & 1803 & 1748 & 1803 & 1748 \\
\hline RMR & 0,089 & 0,086 & 0,060 & 0,056 \\
\hline GFI & 0,710 & 0,822 & 0,972 & 0,976 \\
\hline AGFI & 0,686 & 0,801 & 0,969 & 0,973 \\
\hline NFI & 0,803 & 0,879 & 0,967 & 0,972 \\
\hline TLI & 0,841 & 0,922 & - & - \\
\hline CFI & 0,849 & 0,928 & - & - \\
\hline RMSEA & 0,067 & 0,047 & - & - \\
\hline
\end{tabular}

\section{Modelo de único mediador}

El análisis de único mediador (figura 2) de la macro variable actitudes laborales con relación a las macro variables clima organizacional y comportamiento prosocial, muestra los estimadores de efecto directo e indirecto respectivos.

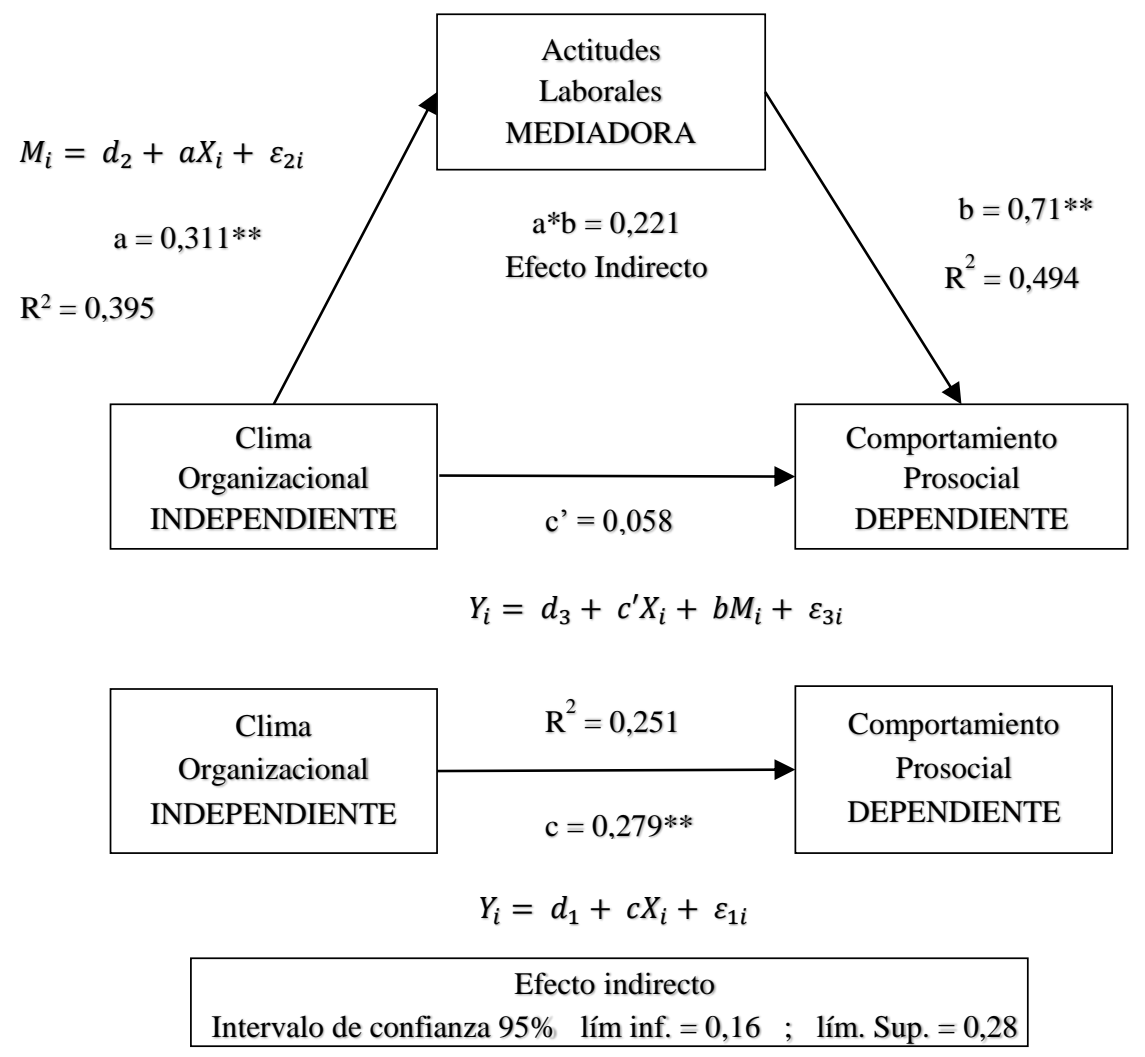

Fig. 2: Mediación simple de clima organizacional, actitudes laborales y comportamiento Prosocial

Se observa en la Fig. 2 que el estimador a) presenta una varianza explicada del $31 \%\left(R^{2}=0,395\right.$ y $t=$ $19,48)$, el estimador b) muestra una varianza explicada del $71 \%\left(R^{2}=0,494\right.$ y $\left.t=16,66\right)$ y el estimador $\left.c\right)$ alcanza un efecto directo con una varianza explicada del $27,9 \%\left(R^{2}=0,251\right.$ y $\left.t=13,96\right)$; todas significativas al $5 \%$. Además, se cumple la relación $c^{\prime}<c \quad(0,058<0,279)$ indicando que el efecto indirecto es significativamente positivo. En consecuencia, se comprueba que en los límites del intervalo de confianza no se encuentra el valor cero (lím. Inf. =0,16 y lím. Sup. =0,28).

Para el análisis de las hipótesis se utilizó las pruebas $\mathrm{F}$ y $\mathrm{T}$, los cuales se compararon con sus respectivos valores estadísticos $\square$ estadístico $=3,841$ y $t$ estadístico $=1,96$; en ambos casos para una muestra infinita con una probabilidad del $5 \%$ de significancia. 
Al verificar $\mathrm{H} 1$ : "que el clima organizacional tiene un efecto directo sobre el comportamiento prosocial, en el contexto de los profesionales de la salud del Guayas, Ecuador", la prueba $F=194,982$ calculada indica que el poder explicativo del modelo en su conjunto es significativo. La prueba $T=13,964$ calculada confirma que el poder explicativo individual de la variables clima organizacional también es significativ0 y dado que el índice $\mathrm{c}=0,279^{\star *}$ significativo, se confirma $\mathrm{H} 1$.

Verificada H2: "que el clima organizacional influyen en forma directa sobre las actitudes laborales, en el contexto de los profesionales de la salud del Guayas, Ecuador", la prueba $F=376,896$ calculada indica que el poder explicativo del modelo en su conjunto es significativo. La prueba $T=19,414$ calculada confirma que el poder explicativo individual del clima organizacional es también significativo y el índice $a=0,311^{* *}$ significativo, se confirma $\mathrm{H} 2$.

Finalmente, al comprobar H3: "Que las actitudes laborales influyen en forma directa y como mediadora entre el clima organizacional y el comportamiento prosocial, en el contexto de los profesionales de la salud del Guayas, Ecuador", la prueba $F=282,805$ calculada indica que el poder explicativo del modelo en su conjunto es significativo. La prueba $\mathrm{T}=16,666$ calculada confirma que el estimador $\mathrm{b}=0,71^{\star *}$ es también significativo.

Por otra parte, el efecto mediador de actitudes laborales que relaciona clima organizacional con comportamiento prosocial se corrobora con la prueba $T=2,759$ calculada, con un índice $c^{\prime}=0,058$ significativo y el efecto indirecto estimado ( $c^{\prime} \leq c$; efecto total - efecto directo; 0,279-0,058) es de 0,221, se confirma que la macro variable actitudes laborales incide directa e indirectamente sobre comportamiento prosocial, en consecuencia, se aprueba $\mathrm{H3}$.

\section{DISCUSIÓN}

La escala original de 44 reactivos y 10 factores de Bustamante et al. (2016) ratificada en la muestra chilena presenta diferencias respecto de la estructura obtenida con la muestra ecuatoriana, puesto que se confirmaron tan solo 29 reactivos que se condensaron en 4 factores. FC1: gestión interna, FC2: estabilidad laboral, FC3: tensión interna y desarrollo profesional, FC4: organización y desempeño.La escala de Actitudes Laborales presentó la misma estructura factorial obtenida en el estudio precedente de Ackfeldt y Wong (2006) con 14 reactivos agrupados en dos dimensiones, FA1: satisfacción laboral y FA2: compromiso afectivo. Por último, el instrumento de medición de comportamiento prosocial adquirió una nueva forma factorial. A diferencia de las 3 dimensiones con 21 ítems detalladas en el estudio de Ackfeldt y Wong (2006), en la actual muestra ecuatoriana persistieron 19 reactivos condensados en 2 dimensiones que describen los factores FP1: cooperación y FP2: calidad y servicio al paciente.

El presente trabajo contribuye a la comprensión del comportamiento prosocial (Kanten, 2014) conformado por las dimensiones endógenas cooperación y calidad y servicio al paciente, las cuales son influidas por las variables latentes exógena de clima organizacional y mediadoras de actitudes laborales que actúan como inductoras directas y significativas. El estudio pone en evidencia que las actitudes laborales (Bülbül, 2014) inciden directamente y además son mediadoras de la incidencia del clima organizacional sobre el comportamiento prosocial. Los hallazgos del presente trabajo constatan, que algunas dimensiones de clima organizacional impactan en forma directa sobre las actitudes laborales como es el caso de gestión interna, confirmando algunos de los resultados de Malhotra y Ackfeldt (2016) y que el factor estabilidad laboral incide de manera directa sobre los dos factores mediadoras de actitudes laborales (Kanten, 2014) y, a través de éstas incide sobre los factores de comportamiento prosocial. En consecuencia, se confirma que el clima organizacional (Bustamante et al, 2016) incide sobre los comportamientos laborales y estimula el comportamiento prosocial (Grant, 2008).

Las ecuaciones estructurales muestran las significativas relaciones encontradas entre los factores latentes mediadores compromiso afectivo con la organización (Choi y Joung, 2017) y satisfacción laboral (Aarons y Sawitzky, 2006) respecto de los comportamientos prosociales altruistas que han sido también reportados (Grant, 2008). Estas relaciones son ratificadas además en el presente trabajo a través del análisis de único mediador, comprobando que la macro variable actitudes laborales incide directa e indirectamente sobre comportamiento prosocial. Resultados equivalentes a los obtenidos por este trabajo, en el ámbito de la salud, fueron reportados por Hsu, et al., (2011) al confirmar que un buen clima organizacional puede incidir directamente sobre las dimensiones de conducta laboral (Liou y Cheng, 2010) y a través de éstas estimular indirectamente las dimensiones de comportamiento prosocial (Kanten, 2014) evidencia que este trabajo, muestra a través del análisis de ecuaciones estructurales y confirma el análisis de un único mediador (MacKinnon y Dwyer, 1993) ratificando el efecto indirecto de la macro variable clima organizacional a través de la macro variable actitudes laborales sobre la macro variable comportamiento prosocial. 


\section{CONCLUSIONES}

De acuerdo con los resultados de este estudio, de su discusión y análisis se alcanzaron cuatro conclusiones principales:

1) Se aprueba $\mathrm{H} 1$ por cuanto los factores de clima organizacional inciden en forma directa sobre los factores de comportamiento prosocial.

2) Se aprueba $\mathrm{H} 2$ dado que los factores clima organizacional efectivamente inciden en forma directa sobre los factores mediadores de actitudes laborales.

2) El análisis de único mediador permite aceptar $\mathrm{H3}$, puesto que las actitudes laborales influyen en forma directa y como mediadora del clima organizacional sobre el comportamiento prosocial, ratificando el hecho que las actitudes laborales transfieren indirectamente parte del efecto del clima organizacional sobre los factores de comportamiento prosocial.

4) Los análisis de primer orden y de segundo orden, permiten afirmar que las macro variables de clima organizacional y de actitudes laborales conforman un sistema interdependiente de efectos que estimula el comportamiento prosocial en el contexto de los profesionales de la salud del Guayas, Ecuador. Finalmente, se sugiere que estas características de influencia mutua entre clima organizacional, actitudes laborales y comportamiento prosocial, puedan ser estudiadas en otros contextos industriales y culturales de latinoamérica.

\section{REFERENCIAS}

Aarons, G. A. y A. C. Sawitzky, Organizational climate partially mediates the effect of culture on work attitudes and staff turnover in mental health services, Administration and Policy in Mental Health and Mental Health Services Research, 33(3), 289 (2006)

Ackfeldt, A. y V. Wong, The Antecedents of Prosocial Service Behaviours: An Empirical Investigation, doi.org/10.1080/02642060600898237, The Service Industries Journal, 26(7), 727-745 (2006)

Arias, W., Conducta prosocial y psicología positiva, Avances en Psicología, 23(1), 37-47 (2015)

Bandalos, D. L. y S. J. Finney, Factor Analysis: Exploratory and Confirmatory. En G.R. Hancock y R. O. Mueller (Eds.), Reviewer's guide to quantitative methods, Routledge, New York (2010)

Baño D.J., E. P. Villacrés, L. F. Arboleda y T. García, Evaluación del clima organizacional del personal docente de las Instituciones de Educación Superior (IES) de la provincia de Chimborazo-Ecuador, doi.org/10.15381/idata.v19i2.12816, Revista Industrial data, 19(2), 59-68 (2016)

Baron, R.M. y D. A. Kenny, The moderator-mediator variable distinction in social psychological research: Conceptual, strategic, and statistical considerations, Journal of personality and social psychology, 51(6), 1173 (1986)

Bernal, I., N. Pedraza y M. Sánchez, El clima organizacional y su relación con la calidad de los servicios públicos de salud: diseño de un modelo teórico, Estudios Gerenciales, 31(134), 8-19 (2015)

Bülbül, A., Social Work Design and Prosocial Organizational Behaviors, Universal Journal of Psychology, 2(2), 47-58 (2014)

Bustamante-Ubilla, M.A., M. Lapo-Maza y M.L. Grandón-Avendaño, Creación de un cuestionario de clima organizacional para hospitales de alta complejidad, Chile, Revista Gerencia y Políticas de Salud, 15(30) (2016)

Chión S., V. Charles, Analítica para la Modelación Estructural (1 ${ }^{\underline{a}}$ Ed.), Perú, Pearson Perú, 239-322 (2016)

Choi, E. K. y H. W. Joung, Employee job satisfaction and customer-oriented behavior: A study of frontline employees in the foodservice industry, Journal of Human Resources in Hospitality \& Tourism, 16(3), 235-251 (2017)

Das, T.V. y C. Vijayalakshmi, Quality of work life-A strategy for good industrial relations, Advances in Management, 6(11), 8 (2013)

Faul, F., E. Erdfelder, A. Buchner y A. G. Lang, Statistical Power Analyses using G*power 3.1: Tests for Correlation and Regression Analyses, Behav Res Methods, 41(4) 1149-1160 (2009)

Freiberg, H.A., J. B. Stover, G. de la Iglesia y M. Fernández, Polychoric and Tetrachoric Correlations In Exploratory and Confirmatory Factorial Studies, Prensa Médica Latinoamericana - ISSN: 1688-4094, Ciencias Psicológicas, VII (2), 151 - 164 (2013)

Grant, A.M., Designing jobs to do good: Dimensions and psychological consequences of prosocial job characteristics, The Journal of Positive Psychology, 3(1), 19-39 (2008)

Henseler, J., C. M. Ringle y M. Sarstedt, A new criterion for assessing discriminant validity in variance-based structural equation modeling, Journal of the academy of marketing science, 43(1) 115-135 (2015) 
Hsu, C., C.W. Chang, H.C. Huang y C.Y. Chiang, The relationships among social capital, organisational commitment and customer-oriented prosocial behaviour of hospital nurses, Journal of Clinical Nursing, 20(9-10) 1383-1392 (2011)

Hyde, P., C. Harris y R. Boaden, Pro-social organisational behaviour of health care workers, The International Journal of Human Resource Management, 24(16), 3115-3130 (2013)

INEC, Instituto Ecuatoriano de Estadisticas y Censos, Compendio Estadístico (2015)

Juárez-Adauta, S., Clima organizacional y satisfacción laboral, Revista Médica del Instituto Mexicano del Seguro Social, 50(3) (2012)

Judd, C.M. y D. Kenny, A. Process analysis: Estimating mediation in treatment evaluations. Evaluation review, 5(5), 602$619(1981)$

Kanten, P., Effect of Quality of Work Life (Qwl) On Proactive and Prosocial Organizational Behaviors: A Research on Health Sector Employees, Suleyman Demirel University Journal of Faculty of Economics \& Administrative Sciences, 19(1) (2014)

Kaplan, D., Structural Equation Modeling, Foundations and Extensions, Thou-sand Oaks, CA, SAGE (2000)

Katz, O. y R. L. Kahn, The social psychology of organizations, New York, Wiley (1966)

Latané, B. y J. M. Darley, The unresponsive bystander: Why doesn't he help? Appleton-Century-Crofts (1970)

Liou, S. R. y C. Y. Cheng, Organisational climate, organisational commitment and intention to leave amongst (2010)

Lloret-Segura, S., A. Ferreres-Traver, A. Hernández-Baeza e I. Tomás-Marco, El análisis factorial exploratorio de los ítems: una guía práctica, revisada y actualizada, Anales de Psicología, 30(3), 1151-1169 (2014)

MacKinnon, D.P. y A.J. Fairchild, Current directions in mediation analysis, Current directions in psychological science, 18(1), 16-20 (2009)

MacKinnon, D.P. y J.H. Dwyer, Estimating mediated effects in prevention studies, Evaluation Review, 17(2), 144-158 (1993)

Malhotra, N. y A.L. Ackfeldt, Internal communication and prosocial service behaviors of front-line employees: Investigating mediating mechanisms, Journal of Business Research, 69(10), 4132-4139 (2016)

Mendoza-Sierra, M.I., A. Orgambídez-Ramos, A.M. Carrasco-González y J.C. León-Jariego, Service climate as a mediator of organizational empowerment in customer-service employees, The Spanish Journal of Psychology, 17 (2014)

Moñivas, A., La Conducta Prosocial, Cuadernos de Trabajo Social, (9), 125- 142 (1996)

Patterson, M., P. Warr y M. West, Organizational climate and company productivity: The role of employee affect and employee level, Journal of Occupational and Organizational Psychology, 77(2), 193-216 (2004)

Smith, R., M. Lagarde y otros ocho autores, Appealing to Altruism: An Alternative Strategy to Address the Health Workforce Crisis in Developing Countries? Journal of Public Health 35(1), 164-170 (2013)

Vandyne, L., L.L. Cummings y J.M. Parks, Extra-role behaviors-in pursuit of construct and definitional clarity (a bridge over muddied waters), Research in Organizational Behavior: An Annual Series of Analytical Essays and Critical Reviews, 17 (17), 215-285 (1995) 